\title{
Research on the injection of new gases in underground methane storage systems in deep aquifers.
}

\author{
PERLA HADDAD ${ }^{1}$, ANTHONY RANCHOU-PEYRUSE ${ }^{2}$,
} FRANCK CASTERAN $^{2}$, MARION GUIGNARD ${ }^{2}$, PIERRE CHIQUET $^{3}$, GUILHEM CAUMETTE ${ }^{3}$, DAVID DEQUIDT ${ }^{4}$ AND PIERRE CÉZAC ${ }^{2}$

${ }^{1}$ ADERA

${ }^{2}$ Université de Pau et des Pays de l'Adour

${ }^{3}$ Téréga

${ }^{4}$ Storengy

Presenting Author: perla.haddad@univ-pau.fr

In order to reduce the use of fossil fuels, biomethane appears to be an effective means of contribution to the energy transition. Oxygen $\left(\mathrm{O}_{2}\right)$ is added to the biomethane during sulfide reoxydation at the outlet of the anaerobic digestion plant. $\mathrm{O}_{2}$ may have impacts during biomethane storage in Underground Gas Storages (UGS) in deep aquifers. Interactions of $\mathrm{O}_{2}$ in the anoxic deep aquifers can modify its biogeochemical conditions. Due to the lack of information, an experimental program is conducted with a view to reproduce in situ conditions and to observe the effects of $\mathrm{O}_{2}$ injection during storage. Results obtained will help define the quality standards required during storages in deep aquifers.

Our simulated aquifer consists of a batch reactor. Three phases are considered: an anoxic groundwater sampled (with autochthonous microorganisms) from a deep aquifer (593 mdeep), rock samples from the reservoir; and a 60 bars gas phase simulating the natural gas with $99 \%$ methane and $1 \% \mathrm{CO}_{2}$. After reaching an equilibrium, $\mathrm{O}_{2}$ at $1 \%$ was injected. Thermodynamic studies, mineral and microbial analysis are conducted.

During the first months of incubation in in situ conditions before $\mathrm{O}_{2}$ injection, the disappearance of sulfate was measured and undoubtedly consumed by the sulfate-reducers microorganisms to produce sulfide. These microorganisms are key players of the microbial communities in deep subsurface. After adding $\mathrm{O}_{2}$, sulfate reduction stopped because of the death of the sulphate-reducers; in addition sulfate reappeared by the reoxidation of the sulfides. Surprisingly, a continuous accumulation of hydrogen appeared after oxygen injection. This phenomenon can be explained by the presence in the microbial community of fermenters producing hydrogen and the disappearance of strict anaerobic hydrogenotrophs, such as certain sulphate-reducers.

The significant arrival of $\mathrm{O}_{2}$ in these reduced ecosystems induces changes in the functioning of microbial communities which in turn interact with the aquifer and the stored gas, influencing the system's established equilibrium. This study shows that far from being anecdotal, these modifications must be taken into account to understand the evolution of gas storages, whether it be methane as on this site or $\mathrm{CO}_{2}$ in the context of geological sequestration. 